\title{
Degradation of the Mode Suppression in Single-Mode Laser Diodes Due to Integrated Optical Amplifiers
}

\author{
Bart Moeyersoon, Student Member, IEEE, Geert Morthier, Senior Member, IEEE, and Mingshan Zhao
}

\begin{abstract}
We show that the side-mode suppression in single-mode laser diodes, such as DFB or distributed Bragg reflector laser diodes, can be reduced by $10 \mathrm{~dB}$ or more if an optical amplifier is integrated with the laser diode. The reduction of the side-mode rejection is due to an increase of spontaneous emission that couples into the side mode, an increase which in turn is due to amplified spontaneous emission originating in the optical amplifier.
\end{abstract}

Index Terms-External feedback, optical communication, semiconductor lasers.

\section{INTRODUCTION}

$\mathbf{T}$ HE SIDE-MODE suppression ratio (SMSR) is one of a number of important laser diode characteristics. Transmitters for high-end optical communication systems typically need to have side-mode rejection over $40 \mathrm{~dB}$. Lower SMSR values may indeed lead to intolerable intensity noise through mode partition noise or to large levels of crosstalk in wavelength division multiplexed (WDM) systems. The power in a side mode typically consists of amplified spontaneous emission (ASE) and its level is determined by the spontaneous emission rate and by the difference between the threshold gain and the mode loss of the side mode (i.e., the mode discrimination). A high side-mode rejection typically requires a low power in the side mode and a high power in the main mode, with the latter normally just requiring a sufficiently high injected current.

Many advanced laser diodes for WDM applications, such as tunable laser diodes or multiwavelength laser diodes, at present are not capable of supplying sufficient output power due to the fact that they include lossy, passive elements such as Bragg sections and combiners. They are therefore often integrated with a semiconductor optical amplifier (SOA) to boost the output power [1], [2]. In a number of previous papers [3], [4], it was shown that in such configurations the ASE from the amplifier couples into the laser diode and may affect the relative intensity noise (RIN) and the linewidth of these laser diodes. Also, the pump lasers in wavelength converters with an integrated pump source ([5] suffer from a similar effect [5].

In this paper, we demonstrate, both theoretically and experimentally, that the ASE from the amplifier also seriously degrades the SMSR of single-mode laser diodes. A degradation of the SMSR of $10 \mathrm{~dB}$ or more is possible due to the presence of an

Manuscript received July 21, 2003; revised October 2, 2003. This work was supported by the Belgian Federal Science Policy Office through the IAP5/18 project.

The authors are with the Department of Information Technology, Ghent University, IMEC, B-9000 Ghent, Belgium (e-mail: morthier@intec.rug.ac.be).

Digital Object Identifier 10.1109/JQE.2003.820840

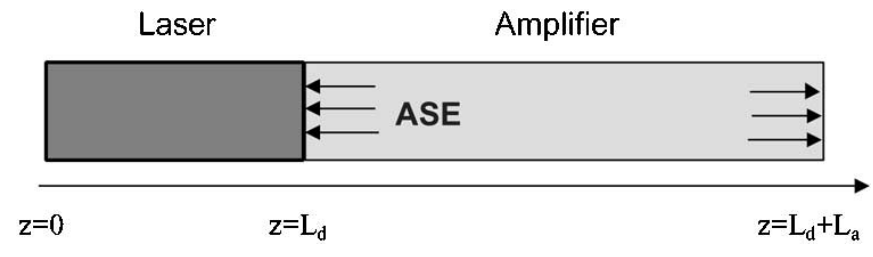

Fig. 1. Schematic structure of a laser diode with an external cavity $(\tau=$ $\left.L_{\text {ext }} / v_{g}\right)$.

integrated SOA (or, equivalently, due to a separate booster amplifier if no isolator is inserted between the laser and amplifier).

A theoretical expression for the increase of the SMSR will be derived in Section II. Numerical and experimental verification of the theory will be given in Sections III and IV, respectively. Section V summarizes the essential results.

\section{THEORETICAL DERIVATION}

The schematic structure of the laser diode integrated with the SOA is shown in Fig. 1. The laser diode is a general multisection DFB or distributed Bragg reflector (DBR) laser diode. A general rate equation for the field of any longitudinal mode in such a laser diode has been derived in a previously published paper [4]. We neglect spatial hole burning and hence assume a uniform carrier density in the active layer of the laser. With $F_{\text {inj }}$ the ASE from the SOA incident into the laser and $F_{c}$ the spontaneous emission generated within the laser, we have

$$
\begin{aligned}
j \frac{\partial \sqrt{1+f}}{\partial t}+v_{g} F_{c} & -j v_{g} F_{\mathrm{inj}} \\
& =\sqrt{1+f}\left\{\Delta \omega+j \frac{1+j \alpha_{H}}{2} G_{l}^{\prime} \delta N\right\} .
\end{aligned}
$$

In this equation, $f$ is the relative variation of the power (smallsignal over steady-state power) and is defined by

$$
\begin{aligned}
\delta R^{ \pm}(z, t) & =R_{0}^{ \pm}(z)\{\sqrt{1+f(t)}-1\} \\
\delta s(z, t) & =s_{0}(z) f(t)
\end{aligned}
$$

where $s(z)$ is the spatial profile of the photon density in the laser cavity and $R^{ \pm}$is the slowly varying, complex amplitudes of forward and backward propagating fields, corresponding to the chosen longitudinal mode. It is assumed that the fields are normalized such that their squared amplitude represents the power.

To derive (1), $R_{0}^{ \pm}$and $N_{0}$ have to be chosen such that they fullfill the static coupled wave equations (i.e., also without spontaneous emission). For a side mode, this means that $N_{0}$ is the threshold carrier density of that side mode. If a main mode is lasing at lower threshold and spontaneous emission is present, 
then the power in the side mode will be ASE and $\delta N$ can be regarded as the deviation from $N_{0}$ due to this ASE.

$G_{l}^{\prime}$ is the modal differential gain (per time unit) of the laser waveguide and $\alpha_{H}$ the linewidth enhancement factor. $\Delta \omega$ is the small-signal variation of the optical frequency of the mode. The Langevin functions $F_{c}$ and $F_{\text {inj }}$ have zero average and the following second-order moments:

$$
\begin{aligned}
& \left\langle v_{g} F_{c}(t) F_{c}^{*}\left(t^{\prime}\right) v_{g}\right\rangle \\
& =(\Gamma g)_{l} n_{\mathrm{sp}, l} v_{g} \frac{K_{z}}{S_{0}} \delta\left(t-t^{\prime}\right) \\
& \left\langle v_{g} F_{\mathrm{inj}}(t) F_{\mathrm{inj}}^{*}\left(t^{\prime}\right) v_{g}\right\rangle \\
& =(\Gamma g)_{a} n_{\mathrm{sp}, a} v_{g} \frac{L_{a}}{L_{d}} \frac{A-1}{\ln (A)} \frac{K_{z}}{S_{0}} \alpha_{r} L_{d} \delta\left(t-t^{\prime}\right) \\
& =(\Gamma g)_{a} n_{\mathrm{sp}, a} v_{g} \frac{L_{a}}{L_{d}} \frac{A-1}{\ln (A)} \frac{K_{z}}{S_{0}} \\
& \quad \times\left\{\begin{array}{c}
\left|R_{0}^{+}\left(L_{d}\right)\right|^{2}\left|t_{2}\right|^{2} \\
\frac{1}{L_{d}} \int_{0}^{L_{d}}\left[\left|R_{0}^{+}\right|^{2}+\left|R_{0}^{-}\right|^{2}\right] d z
\end{array}\right\} \delta\left(t-t^{\prime}\right)
\end{aligned}
$$

where $L_{d}$ is the laser diode length and $S_{0}$ is the number of photons in the laser cavity. $(\Gamma g)_{l}, n_{\mathrm{sp}, 1}, \alpha_{l}$ and $(\Gamma g)_{a}, n_{\mathrm{sp}, \mathrm{a}}, \alpha_{a}$ are the modal gain, inversion factor, and internal loss of the laser and the amplifier, respectively. $A$ is the amplification of the amplifier, $K_{z}$ is the longitudinal Petermann factor, and $\alpha_{r} L_{d}$ is the extraction efficiency of the laser facet [4].

Equation (1) is a nonlinear differential equation, which we can rewrite also as

$$
\begin{aligned}
\frac{\partial f}{\partial t}=2 j\left[v_{g} F_{c}\right. & \left.-j v_{g} F_{\mathrm{inj}}\right] \sqrt{1+f} \\
& -2 j(1+f)\left\{\Delta \omega+j \frac{1+j \alpha_{H}}{2} G_{l}^{\prime} \delta N\right\} .
\end{aligned}
$$

The first term on the right-hand side (r.h.s.) gives rise to the dc contribution of the noise, and this term can easily be calculated using a perturbation approach. Taking the real part of (5) and neglecting $f, \Delta \omega$, and $\delta N$ in the r.h.s., gives the following solution for $f$ :

$$
f(t)=R e \int_{-\infty}^{t}\left[2 j v_{g} F_{c}\left(t^{\prime}\right)+2 v_{g} F_{\mathrm{inj}}\left(t^{\prime}\right)\right] d t^{\prime} .
$$

We then substitute this expression in (5), linearize in $f$, and take the statistical (or time) average, i.e.,

$$
\begin{aligned}
\left\langle\operatorname{Re}\left[2 j v_{g} F_{c}(t)+2 v_{g} F_{\mathrm{inj}}(t)\right] f(t)\right\rangle \\
=4 v_{g}^{2}\left\langle\operatorname{Re}\left[j F_{c}(t)+F_{\mathrm{inj}}(t)\right]\right. \\
\left.\quad \times \operatorname{Re} \int_{-\infty}^{t}\left[j F_{c}\left(t^{\prime}\right)+F_{\mathrm{inj}}\left(t^{\prime}\right)\right] d t^{\prime}\right\rangle \\
=\frac{(\Gamma g)_{l} n_{\mathrm{sp}, 1} v_{g} K_{z}}{S_{0}}+\frac{(\Gamma g)_{a} n_{\mathrm{sp}, \mathrm{a}} v_{g} K_{z}}{S_{0}} \frac{L_{a}}{L_{d}} \frac{A-1}{\ln (A)} \alpha_{r} L_{d}
\end{aligned}
$$

Hence, when linearizing equation (5) and separating it into real and imaginary parts, the equation for $f$ should be written as

$$
\begin{aligned}
\frac{\partial f}{\partial t}=2 v_{g}\left[\operatorname{Re}\left(F_{\mathrm{inj}}\right)-\operatorname{Im}\left(F_{c}\right)\right]+G_{l}^{\prime} \delta N \\
+\frac{R_{\mathrm{sp}, l}+R_{\mathrm{sp}, a} \frac{L_{a}}{L_{d}} \frac{A-1}{\ln (A)} \alpha_{r} L_{d}}{S_{0}}
\end{aligned}
$$

where $R_{\mathrm{sp}, l}$ and $R_{\mathrm{sp}, a}$ are the spontaneous emission rate per time unit for the laser and amplifier, respectively. From now on, we ignore the noise and concentrate on the spontaneous emission that couples into the mode. Equation (8) can then be expressed as

$$
\frac{\partial S}{\partial t}=G_{l}^{\prime} \delta N S_{0}+R_{\mathrm{sp}, l}+R_{\mathrm{sp}, a} \frac{L_{a}}{L_{d}} \frac{A-1}{\ln (A)} \alpha_{r} L_{d} .
$$

For a side mode, as mentioned above, $\delta N$ is the difference between the actual carrier density in the presence of ASE and the threshold carrier density for that mode and hence $G_{l}^{\prime} \delta N=$ $G_{\text {th }}-1 / \tau_{p}$ where $\tau_{p}$ is the photon lifetime for the side mode and $G_{\text {th }}$ the threshold gain of the main mode. The static solution of (9) therefore can be written as

$$
S_{0}=\frac{R_{\mathrm{sp}, l}+R_{\mathrm{sp}, a} \frac{L_{a}}{L_{d}} \frac{A-1}{\ln (A)} \alpha_{r} L_{d}}{1 / \tau_{p}-G_{\mathrm{th}}}
$$

where $S_{0}$ is the power in the side mode. This power is obviously increased due to the presence of an SOA and the increase is with a factor

$$
\frac{S_{0}^{\mathrm{SOA}}}{S_{0}^{\mathrm{noSOA}}}=1+\frac{L_{a}}{L_{d}} \frac{A-1}{\ln (A)} \alpha_{r} L_{d} \frac{(\Gamma g)_{a} n_{\mathrm{sp}, a}}{(\Gamma g)_{l} n_{\mathrm{sp}, l}}
$$

i.e., exactly the same factor that was found for the linewidth increase due to the ASE of an integrated SOA; this can be attributed to the fact that both SMSR and linewidth depend on the total spontaneous emission that couples into a laser mode. This factor is of course the factor by which the side mode suppression, measured at the facet $z=0$, decreases.

\section{NUMERICAL RESULTS}

As a first verification of the theoretical results obtained above, we performed some numerical simulations using the commercially available VPI software [6]. The following parameters are applicable for the structure from Fig. 1: the laser was a quarterwave-shifted DFB laser with a length of $L_{d}=400 \mu \mathrm{m}$ and a normalized coupling coefficient $\kappa L_{d}=2$; the length of the SOA was $L_{a}=350 \mu \mathrm{m}$ and its amplification $A=16 \mathrm{~dB}$. Both the laser and SOA had an inversion factor $n_{\mathrm{sp}}=2$.

Fig. 2 shows the obtained variation of the side-mode suppression for increasing levels of ASE power $P_{\text {ase }}$ injected into the laser and this for three different laser output powers of 0.6, 2, and $5 \mathrm{~mW}$, respectively. These values correspond to the output power of the solitary laser, without the presence of an amplifier. For clarity, we would also like to note that the numerical values of $P_{\text {ase }}$ in this graph correspond to the total ASE power emitted by the SOA when no laser would be present; this is different from the ASE coupling into the side mode.

The total emitted ASE power from the SOA is proportional with $R_{\mathrm{sp}, a}(A-1) / \ln A$. However, instead of varying the amplification to vary the ASE injected into the laser, we have applied 


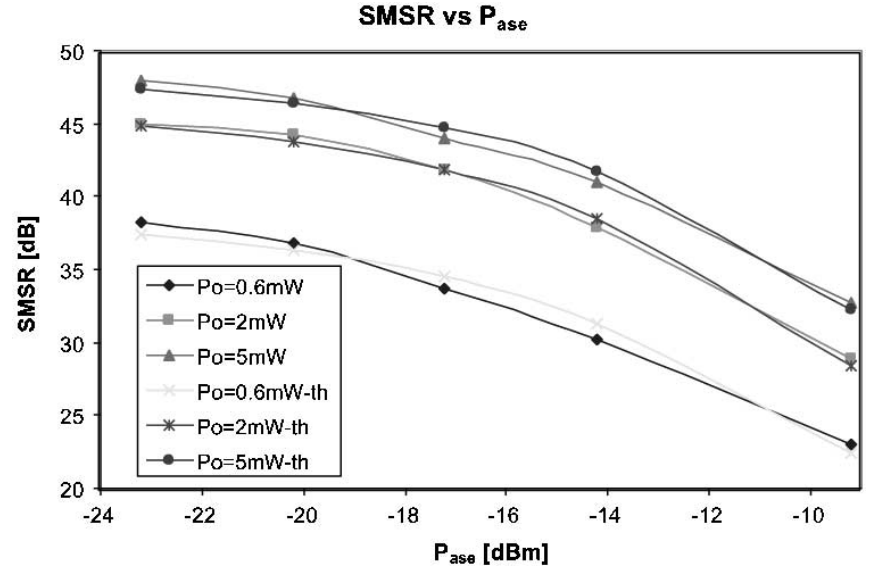

Fig. 2. Calculated SMSR values and theoretical fits versus $P_{\text {ase }}$ for three different laser output powers of $0.6,2$, and $5 \mathrm{~mW}$, respectively $\left[P_{o}\right.$ is the output power of the solitary laser and -th indicates the fitted results obtained from (12)].

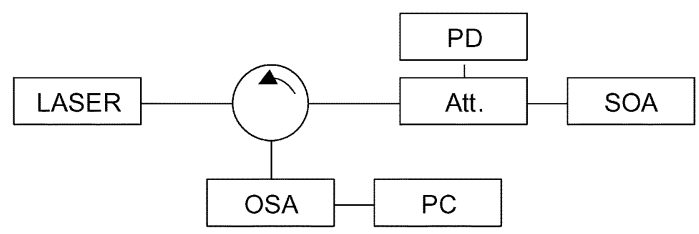

Fig. 3. Schematic of the measurement setup. OSA: optical spectrum analyzer; Att.: attenuator; PD: photodetector.

various levels of attenuation on the ASE emitted from the SOA, while keeping the current through the SOA constant.

From Fig. 2, one can clearly see the degradation of the SMSR for increasing $P_{\text {ase }}$. For an increase of $P_{\text {ase }}$ of $14 \mathrm{~dB}$ (from -23 to $-9 \mathrm{dBm}$ ), the decrease of the SMSR is 15,16 , and $15 \mathrm{~dB}$ for laser output powers of $0.6,2$, and $5 \mathrm{~mW}$, respectively.

The numerically obtained results were fitted to the following expression for the SMSR, which was derived from (11) by taking the attenuation $a$, by which the ASE emitted by the amplifier is attenuated (proportional to $(\Gamma g)_{a} n_{\mathrm{sp}, a}(A-1) / \ln A$ ), into account and by grouping the remaining parameters into a proportionality factor $\eta$ :

$$
\frac{\mathrm{SMSR}^{\mathrm{SOA}}}{\mathrm{SMSR}^{\mathrm{noSOA}}}=\left[1+\frac{\eta}{a} \frac{A-1}{\ln (A)}\right]^{-1}
$$

The theoretical fits obtained from (12) are also shown (using -th in the legend) in Fig. 2, and we see good qualitative agreement between the theoretical and simulated results.

\section{EXPERIMENTAL RESULTS}

To further verify our theory, we performed some measurements. The measurement setup is shown in Fig. 3. The laser was a packaged tunable three section DBR laser with an AR-coated facet and without isolator in the package. The SMSR of this laser is rather low (only $35 \mathrm{~dB}$ ), but this does not pose a problem, since we are mainly interested in the decrease of the SMSR when ASE is injected into the laser. We used a polarization-independent SOA with a center wavelength of $1550 \mathrm{~nm}$ and an ASE power of $-7.5 \mathrm{dBm}$ as ASE source. The ASE was injected into the laser using a circulator and the injected $P_{\text {ase }}$ was varied using

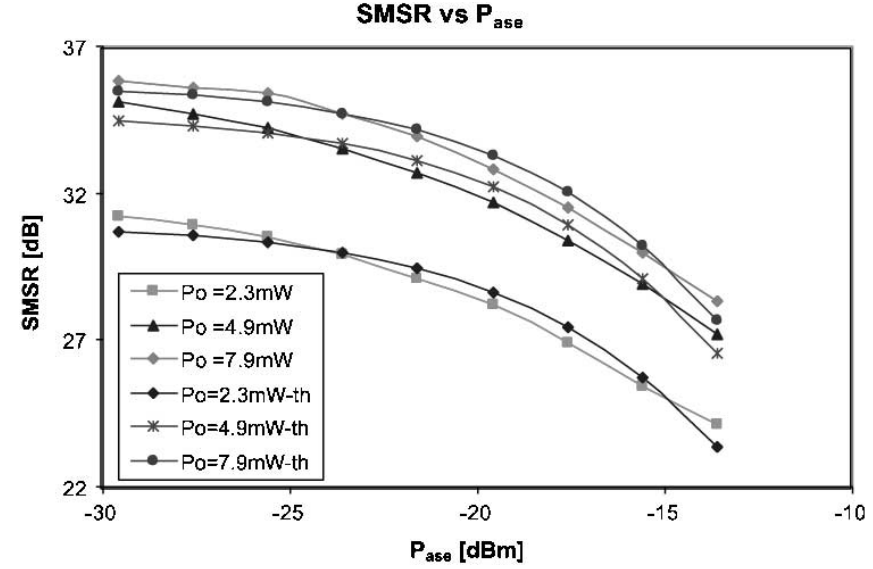

Fig. 4. Measured SMSR values and theoretical fits versus $P_{\text {ase }}$ for three different laser output powers of $2.3,4.9$, and $7.9 \mathrm{~mW}$, respectively $\left[P_{o}\right.$ is the output power of the solitary laser, and -th indicates the fitted results obtained from (12)].

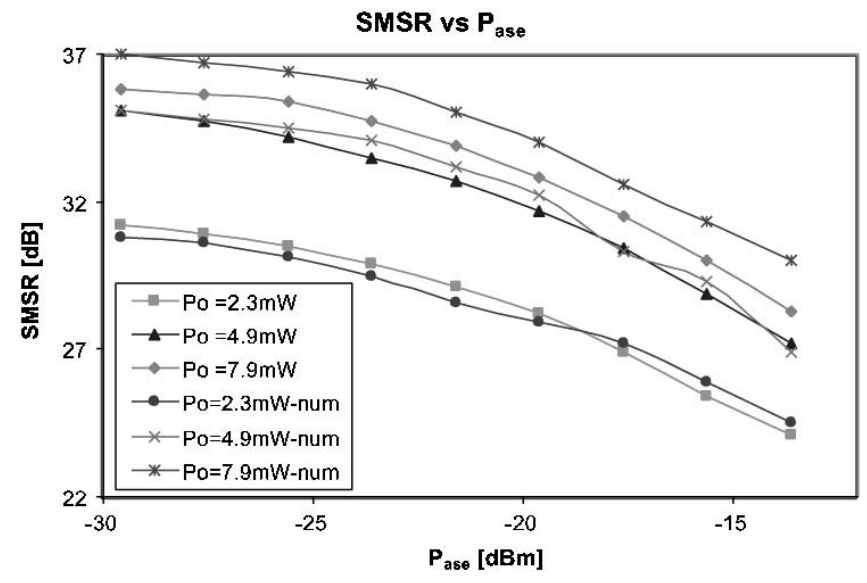

Fig. 5. Measured SMSR values and numerically calculated values versus $P_{\text {ase }}$ for three different laser output powers of $2.3,4.9$, and $7.9 \mathrm{~mW}$, respectively $\left(P_{o}\right.$ is the output power of the solitary laser, -num indicates the numerically calculated results).

an attenuator. The output of the aggregate system was monitored using a computer-controlled optical spectrum analyzer.

In this configuration, the amplifier will not be saturated by the laser light, which would be the case for an integrated amplifier. Nevertheless, we will be able to investigate the influence of (different levels of) ASE coupled into a laser diode on the laser performance.

Fig. 4 shows the measured variation of the SMSR for increasing levels of emittted ASE power $P_{\text {ase }}$, and again the theoretical fits obtained from (12) are also shown (using -th in the legend), and this for three different laser output powers of 2.3, 4.9 , and $7.9 \mathrm{~mW}$, respectively (again, these values indicate the output power of the laser when no amplifier would be present). The amplification of the SOA was $16 \mathrm{~dB}$. Again we see the degradation of the SMSR. When the emitted ASE power increases $16 \mathrm{~dB}$ (from -29.5 to $-13.5 \mathrm{dBm}$ ), the SMSR decreases 7,8 , and $7.5 \mathrm{~dB}$ for laser output powers of $2.3,4.9$, and $7.9 \mathrm{~mW}$, respectively. We also see good qualitative agreement between the theoretical and measured results.

Fig. 5 again shows the experimental results, now compared with numerically calculated values. For this calculation, the 


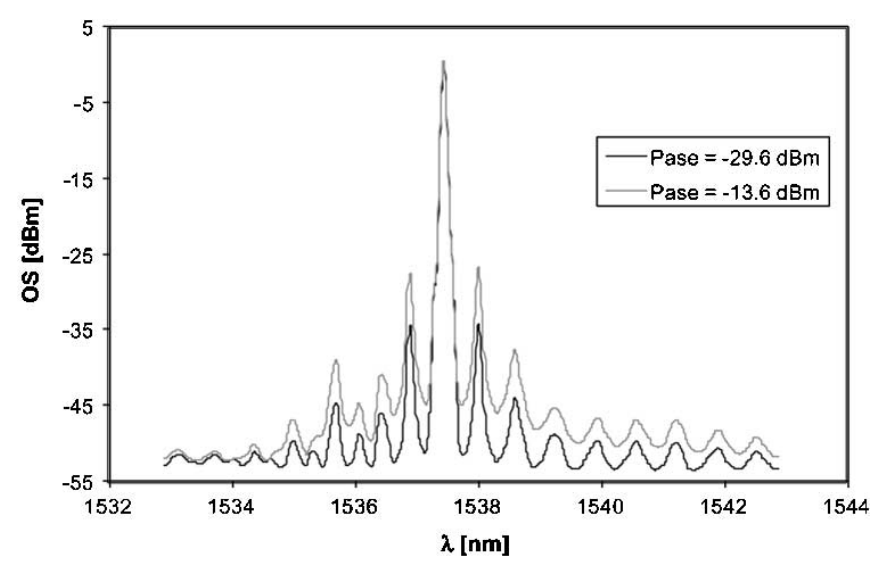

Fig. 6. Measured optical spectrum (OS) for two different levels of $P_{\text {ase }}$ (-29.6 and $-13.6 \mathrm{dBm}$, respectively).

parameters of the laser model were adapted to match as well as possible with those of the laser used in the experiments. Since we do not know all of the parameters of our laser that are required for the numerical model [6], and furthermore do not know the parameter values related to the coupling of the laser and the amplifier, it is difficult to obtain good agreement between numerical and experimental values for all laser output powers. On the contrary, (12) yields an easy and reliable means to correctly describe the experimental results, without having to know all of the parameters involved.

Fig. 6 shows the optical spectrum for two different levels of $P_{\text {ase }}(-29.6$ and $-13.6 \mathrm{dBm}$, respectively). The peaks of the spectra are overlapping and the decrease of the SMSR can clearly be seen.

\section{CONCLUSION}

We have shown theoretically, numerically, and experimentally that an SOA integrated with a laser diode degrades the side-mode suppression of that laser diode significantly. This is due to an increase of the ASE coupled in the side mode and not due to any change in the mode discrimination. As a result, the increased side-mode power will not result in a deterioriation of the dynamic single-mode stability or to increased mode partition noise. It may, however, lead to increased crosstalk in WDM systems.

\section{REFERENCES}

[1] B. Mason, J. Barton, G. A. Fish, L. A. Coldren, and S. P. Denbaars, "Design of sampled grating DBR lasers with integrated semiconductor optical amplifiers," IEEE Photon. Technol. Lett., vol. 12, pp. 762-764, July 2000.

[2] K. Kundo, K. Yashiki, T. Sasaki, Y. Yokoyama, K. Hamamoto, T. Morimoto, and M. Yamaguchi, "1.55- $\mu \mathrm{m}$ wavelength-selectable microarray DFB-LD's with monolithically integrated MMI combiner, SOA and EA-modulator," IEEE Photon. Technol. Lett., vol. 12, pp. 242-244, Mar. 2000.
[3] E. Berglind and O. Nilsson, "Laser linewidth broadening caused by a laser amplifier," IEEE Photon. Technol. Lett., vol. 3, pp. 442-444, May 1991.

[4] G. Morthier and B. Moeyersoon, "Intensity noise and linewidth of laser diodes with integrated semiconductor optical amplifier," IEEE Photon. Technol. Lett., vol. 14, pp. 1644-1646, Dec. 2002.

[5] L. H. Spiekman, J. M. Wiesenfeld, U. Koren, B. I. Miller, and M. D. Chien, "All-optical Mach-Zehnder wavelength converter with monolithically integrated preamplifiers," IEEE Photon. Technol. Lett., vol. 10, pp. 1115-1117, Aug. 1998.

[6] Componentmaker [Online]. Available: http://www.virtualphotonics. $\mathrm{com} /$

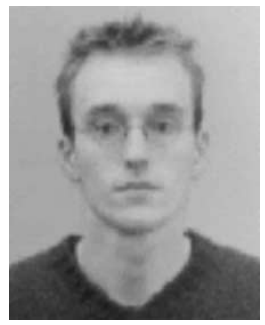

Bart Moeyersoon (S'97) was born in Aalst, Belgium, in 1976. He received the degree in electrical engineering from the University of Ghent, Ghent, Belgium, in 1999 and is currently working toward the Ph.D. degree in electrical engineering at the same university.

His research interests include tunable lasers and wavelength-division-multiplexed networks.

Mr. Moeyersoon is a member of the IEEE Lasers and Electro-Optics Society.

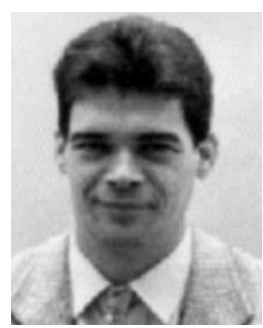

Geert Morthier (M'93-SM'01) received the degree in electrical engineering and the Ph.D. degree from the University of Ghent, Ghent, Belgium, in 1987 and 1991, respectively.

Since 1991, he has been a member of the permanent staff of IMEC, University of Ghent. His main interests are in the modeling and characterization of optoelectronic components. He has authored or coauthored approximately 100 papers in the field. $\mathrm{He}$ is also one of the two authors of the Handbook of Distributed Feedback Laser (Boston, MA: Artech House, 1997) and coeditor of the book How to Model and Measure Photonic Components: Experience from a European Project (Berlin, Germany: Springer-Verlag, 1998). From 1998 through the end of 1999, he was the project manager of the ACTS project ACTUAL dealing with the control of widely tunable laser diodes, and since 2001 he has been the project manager of the IST project NEWTON on new widely tunable lasers. In 2001, he was appointed a part-time Professor at the University of Ghent.

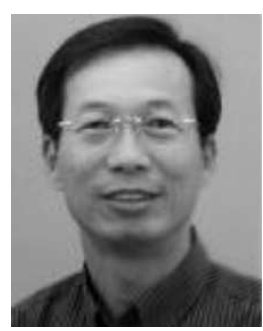

Mingshan Zhao received the B.S. degree in physics from Qufu Normal University, Shandong, China, in 1982, the M.S. degree in condensed matter physics from Beijing Normal University, Beijing, China, in 1986, and the Ph.D. degree in electrical engineering from the University of Ghent, Ghent, Belgium, in 2003.

From 1986 to 1998 , he was involved with optical components and systems in the Laser Institute, Qufu Normal University. In 1998, he joined the Department of Information Technology, University of Ghent. His current interests include all-optical signal processing and wavelength division multiplexing. He has authored or coauthored over 40 papers. 